ISBN 978-93-84468-80-4

International Conference on Recent Trends in Engineering and Technology

(RTET-2016)

Pattaya (Thailand) Dec. 14-16, 2016

\title{
Simulation of the Apply Integral Control and Bias Estimator on Proximate Time Optimal Servo System
}

\author{
Reyhaneh Azad ${ }^{1}$, Vala Saleh ${ }^{2}$, Syed khaleel Ahmed ${ }^{3}$, Mohammadreza Siyahtiri ${ }^{4}$ \\ ${ }^{1,2,3}$ Faculty of Engineering, University Tenaga Nasional, Malaysia, \{azadreyhaneh,valasaleh $\} @$ yahoo.com, \\ syedkhaleel@uniten.edu.my \\ ${ }^{4}$ Faculty of Engineering and Built Environment,The National University of Malaysia \\ Malaysia,m.siyahtiri@yahoo.com
}

\begin{abstract}
Time optimal performance is the main objective of hard disk drive (HDD) servo system to pick and place read/write $(R / W)$ head from an initial track to a final. Time optimal control (TOC) has the fastest response in time seeking mode. But due to the chattering problem, it is not a well-established controller in a practical stage. So, proximate time optimal servomechanism (PTOS) suggested as a most common near time optimal technique to solve the TOC problems. However, the rise time of PTOS is more than TOC. This paper exposes the correct deployment of PTOS with two methods of integral control and bias estimator for a double integrator rigid body in MATLB to reduce time response and eliminate steady state error.
\end{abstract}

Keywords: Component; hard disk drive servosystem, proximate time optimal control, Integral control, bias estimator

\section{Introduction}

Hard disk drive (HDD) plays an important role in data-storage medium for most of data processing systems. It stores and retrieves data by using a magnetic Read/Write (R/W) head. HDD servo system is a control system that places the head over a given track and repositions that from initial track to final one [1]. In hard disk drive (HDD) servo system control, the ability of the system to quickly tracking a desired point is one of the main performance criteria. TOC is first proposed as a way to increase the speed of head positioning servo system and decrease the time to track seeking in HDDs. But, it is not feasible in applications because of sensitivity to the parameter change [2], input disturbances [3] and accordingly, control chatter [4]. As a result, a near time-optimal response must be desired [5].

There are many near TOC method such as PTOS, variable structure sliding mode control, adaptive control, linear quadratic Gaussian control (LQG), mode switching control, etc [6]. PTOS is a most popular one, which has a simple and good reputation algorithm for control of hard disk drive servo systems [7-10]. It combines variable structure control (VSC) with PD for keeping near time optimality in the presence of input disturbances and parameter variation [11]. Many reforms have been carried out on PTOS. Dhanda et al. improved the velocity error by defining two free variables in control design [11]. Kalyon et al. offered near linear action close to the reference point apart from small and quickly declining affects of the nonlinear condition, and, a nonlinear behavior of the servo within a narrow band in the proximity of the switching curve [12]. Most of this correction improves the PTOS performance. However, PTOS rise time is more than TOC. To reduce settling time and eliminate steady state error in PTOS algorithm higher gain at lower frequency is needed that is possible by using integral control.

In this paper, two methods to use Integrator for rejection of input disturbance, which are the PTOS with integral such that the output of integrator is a new state, control and PTOS with bias estimator are simulated in SIMULINK environment of MATLB. 
This work continues as follow. A Preliminary on the parameters and models needed for the implementation of the present work reviewed in section II. The simulation is described in section III while the results are presented and discussed in section IV. Concluding remarks are made in section V.

\section{Preliminaries}

\subsection{System Modeling and Control Law}

In this work, VCM actuator is assumed as a double integrator, $\frac{a}{s^{2}}$, where $a=6.3 \times 10^{7}$ is the acceleration constant. For this model, the PTOS control signal is

$$
\mathrm{u}=\mathrm{U}_{\mathrm{m}} \mathrm{sat}\left\{\mathrm{k}_{2} \frac{\mathrm{f}_{\mathrm{PTOS}}-\mathrm{x}_{2}}{\mathrm{U}_{\mathrm{m}}}\right\}
$$

Where $x_{2}$ is negative of derivative of the error signal and

$$
\mathrm{f}_{\text {РтоS }}= \begin{cases}\operatorname{sgn}(\mathrm{e})\left(\sqrt{2 \mathrm{a} \alpha \mathrm{U}_{\mathrm{m}}|\mathrm{e}|}-\frac{\mathrm{U}_{\mathrm{m}}}{\mathrm{k}_{2}},\right. & |\mathrm{e}|>\mathrm{e}_{\mathrm{l}} \\ \frac{\mathrm{k}_{1}}{\mathrm{k}_{2}} \mathrm{e}, & |\mathrm{e}| \leq \mathrm{e}_{\mathrm{l}}\end{cases}
$$

Where, $\boldsymbol{\alpha}(\boldsymbol{\alpha}<\mathbf{1})$ is acceleration discount factor, $\boldsymbol{k}_{\mathbf{1}}$ and $\boldsymbol{k}_{\mathbf{2}}$ are State feedback gains and $\boldsymbol{e}_{\boldsymbol{l}}=\mathbf{2 0}$ tracks is upper limit of position error and sat is saturation function.

\subsection{State Estimator}

As regards the control designs needs a full knowledge about the system states and the head position y is the unique measurable signal for state feedback control, it is essential to estimate the system states via a state estimator. However, it is not the objective of this work. Hence, a brief debate on the estimator design is presented.

Double integrator in state space model as

$$
\dot{\mathrm{x}}=\mathrm{Ax}+\mathrm{Bu}, \mathrm{y}=\mathrm{Cx}
$$

Where $\mathrm{x}(\mathrm{t})$ is the state vector $\left[\begin{array}{ll}\mathrm{x}_{1} & \mathrm{x}_{2}\end{array}\right]^{\mathrm{T}}$ consists of position $\left(\mathrm{x}_{1}\right)$ and velocity $\left(\mathrm{x}_{2}\right), \mathrm{u}(\mathrm{t})$ is input of VCM, $\mathrm{y}(\mathrm{t})$ is output of VCM and $A=\left[\begin{array}{ll}0 & 1 \\ 0 & 0\end{array}\right], B=\left[\begin{array}{l}0 \\ a\end{array}\right], C=\left[\begin{array}{ll}1 & 0\end{array}\right]$.

The state estimator formulates as

$$
\dot{\hat{x}}=A \hat{x}+B u+L(y-C \hat{x})
$$

Where $\hat{\mathrm{x}}$ is the estimate of the state vector $\mathrm{x}$ and the observer gain, $\mathrm{L}$, can be calculated via Ackerman's formula and pole placement by choosing the poles of the state estimator be ten times faster than the poles of the system. In our case, the natural frequency and damping ratio of the closed loop system sI-(A-BK) are achieved such that $\omega_{\mathrm{n}}=2.74, \zeta=0.74$ and settling time $\left(\mathrm{T}_{\mathrm{s}}\right)$ of system is $\mathrm{T}_{\mathrm{s}} \cong 2$.

Hence, the desired equation for estimator is

$$
s^{2}+40 s+730=0
$$

Using Ackermann's formula

$$
\mathrm{L}=\mathrm{q}(\mathrm{A}) \mathrm{s}_{\mathrm{o}}^{-1}\left[\begin{array}{ll}
0 & 1
\end{array}\right]^{\mathrm{T}}
$$


Where $\mathbf{q}(\mathbf{A})=\mathbf{A}^{\mathbf{2}}+\mathbf{2} \boldsymbol{\zeta} \boldsymbol{\omega}_{\mathbf{n}} \mathbf{A}+\boldsymbol{\omega}_{\mathbf{n}}{ }^{2}$ and $\mathbf{s}_{\mathbf{o}}=\left[\begin{array}{ll}\mathbf{B} & \mathbf{B A}\end{array}\right]$. So, matrix L is achieved as

$$
L=\left[\begin{array}{r}
40 / \mathrm{a} \\
730 / \mathrm{a}
\end{array}\right]
$$

\subsection{Design of Integral Control for Steady State Error Specification}

Based on (5) the feedback gain can be calculated directly as $\mathrm{k}=\left[\begin{array}{ll}\mathrm{k}_{1} & \mathrm{k}_{2}\end{array}\right]=\left[\begin{array}{lll}730 & 40\end{array}\right]$. Hence the compensated system models in state space as

$$
\begin{gathered}
\dot{x}(t)=\left[\begin{array}{cc}
0 & 1 \\
-730 & -40
\end{array}\right] x(t)+\left[\begin{array}{l}
0 \\
a
\end{array}\right] r(t) \\
y(t)=\left[\begin{array}{ll}
1 & 0
\end{array}\right] x(t)
\end{gathered}
$$

And the characteristic equation of the compensated system using integral controller by assuming third pole at $\mathrm{s}=10 * \zeta \omega_{\mathrm{n}}=20$, achieves as

$$
s^{3}+24 s^{2}+87.5 s+15=0
$$

Hence the following coefficient is obtained

$$
\mathrm{k}_{1}=\frac{87.5}{\mathrm{a}}, \mathrm{k}_{2}=\frac{24}{\mathrm{a}} \text { and } \mathrm{k}_{\mathrm{e}}=\frac{15}{\mathrm{a}}
$$

\section{Simulation}

The aforementioned integral control has been applied to the PTOS controller through two methods.

- Add integral control such that the output of integrator is a new state.

- Add bias estimator

This section will give the construction and implementation of the PTOS with Two integral controllers to decreases PTOS weakness and control of the HDD servo system in MATLAB/SIMULINK environment.

\subsection{PTOS with Integral Control}

To evaluate the first way, an integrator is added to feedback path. Fig. 1 is the simulation of PTOS with integral control. 


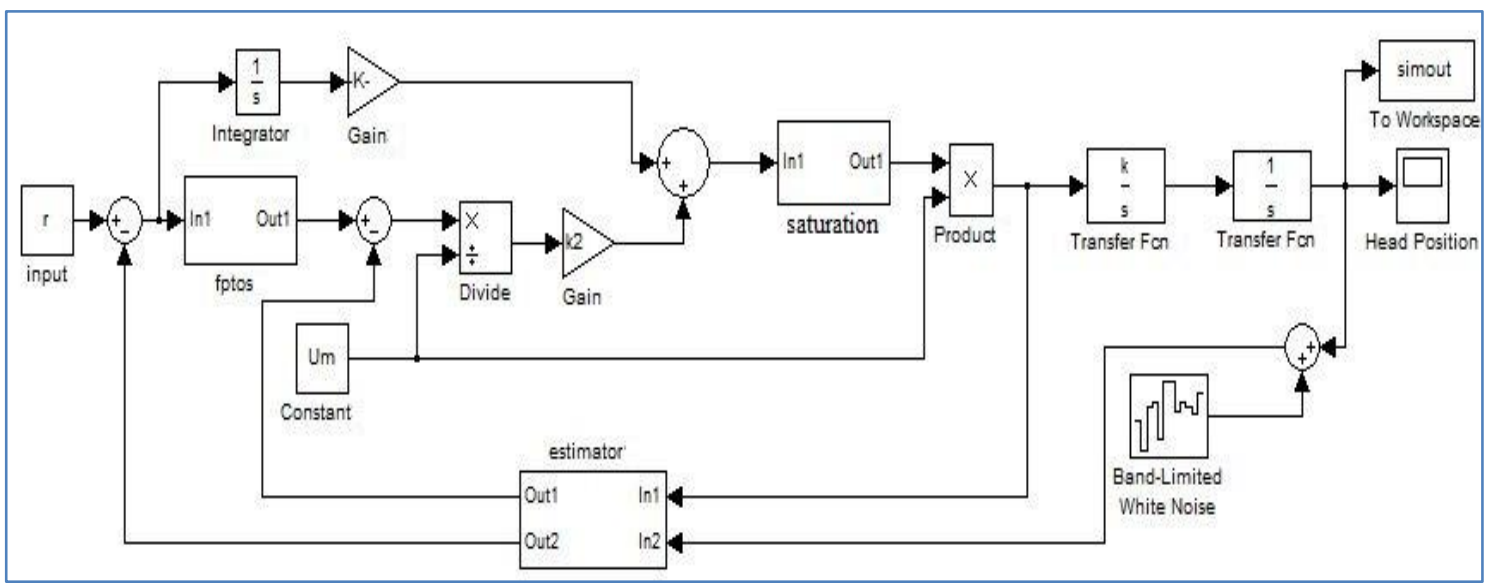

Fig 1. PTOS with integral control: block diagram

The noise is added to validate the effect of integral control on disturbance rejection.

As you see

$$
\dot{\mathrm{X}}_{\mathrm{I}}=\mathrm{X}_{\mathrm{I}}+\mathrm{y}_{\mathrm{r}}-\mathrm{y}
$$

\subsection{PTOS with Bias Estimator}

The second of two methods to nullify the effect of disturbance is adding the open loop state space model such that input bias is a new state. To realize the PTOS with bias estimator, the bias estimator is implemented in MATLAB/SIMULINK. Fig.2 illustrates the simulated block diagram of bias estimator. As you can see it has 2 inputs and 3 outputs. The inputs are exactly same as before. Out 1 and Out 2 which are presented in Fig. 2 are states to make the control signal using the feedback law, Out 3 is estimated by the adding observer but it could not controlled via feedback.

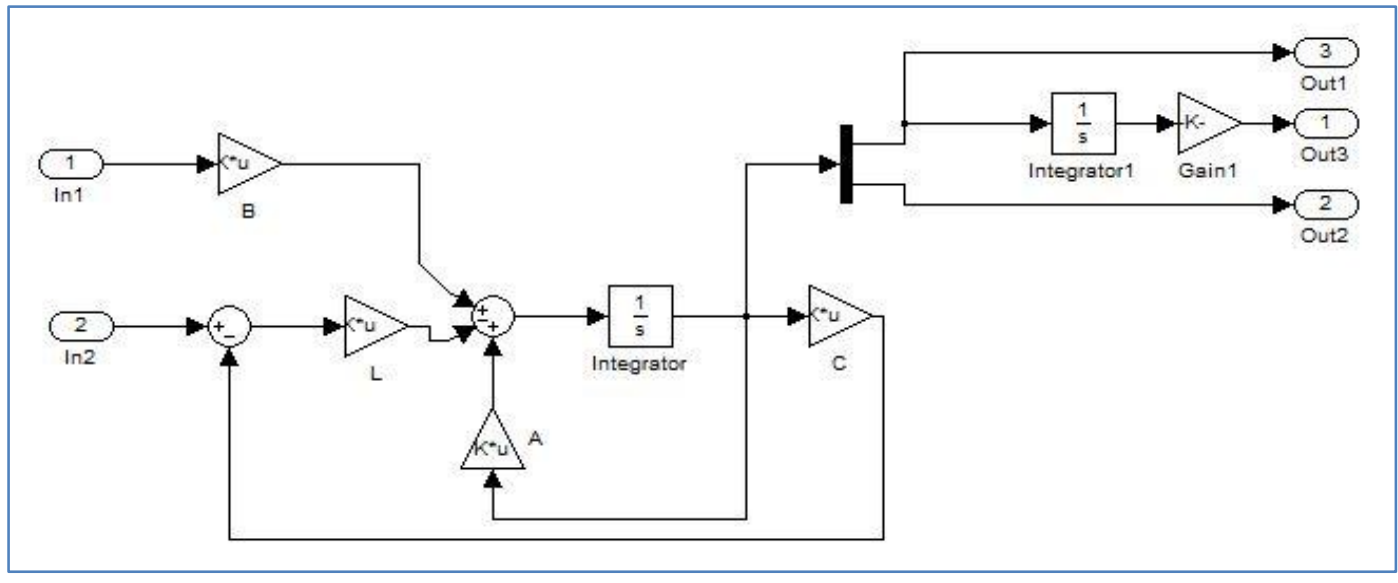

Fig. 2 Bias estimator: block diagram

Block diagram of Fig. 2 is used in Fig.3 as estimator block.

By using this method the order of the estimator increases by 1 . When the order of system increased, we can meet the steady state error specification. 


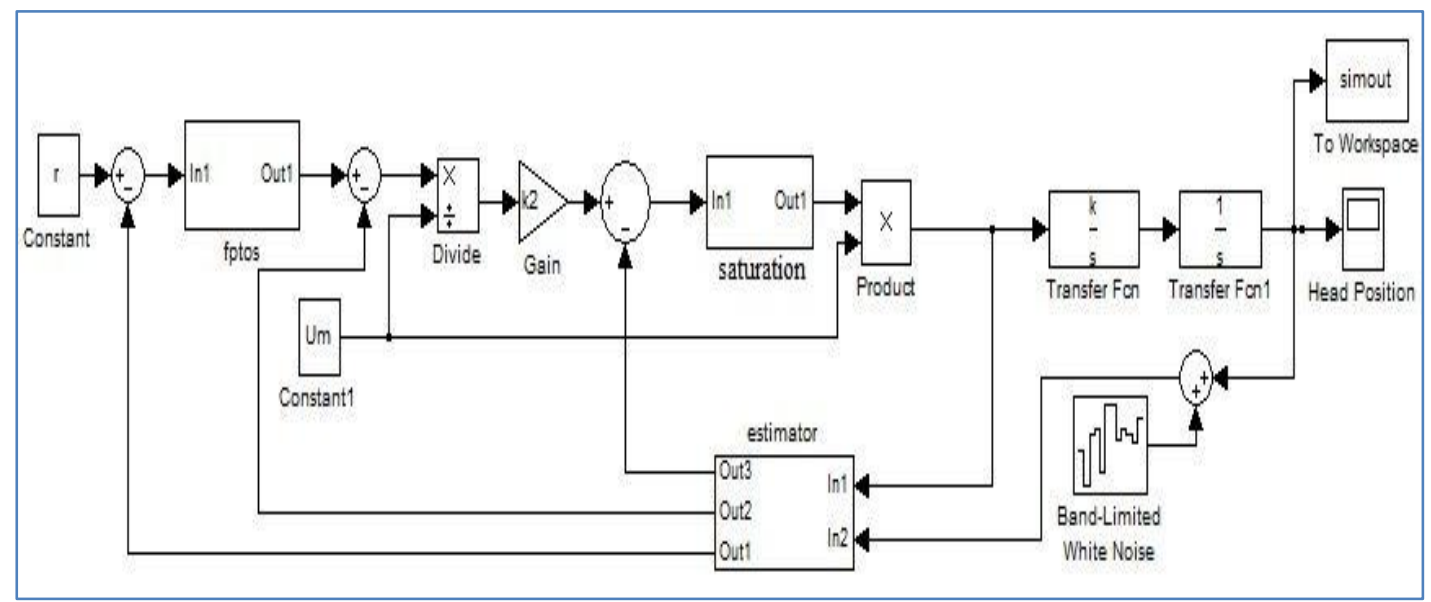

Fig 3. PTOS with bias estimator: block diagram

\section{Results and Discussion}

Simulations are conducted on the system to compare the performances of the PTOS with integral control and PTOS with bias estimator. The responses of these two servo systems and comparison among them and PTOS are demonstrated in Fig.4, 5 and 6, respectively. These figures emphasize the improvement of settling time of PTOS with integral control and bias estimator compared to PTOS. Fig.4 shows seek response of PTOS controller with state estimator and integral control in presence of the noise. As can be seen the seek time is about 5 milliseconds.

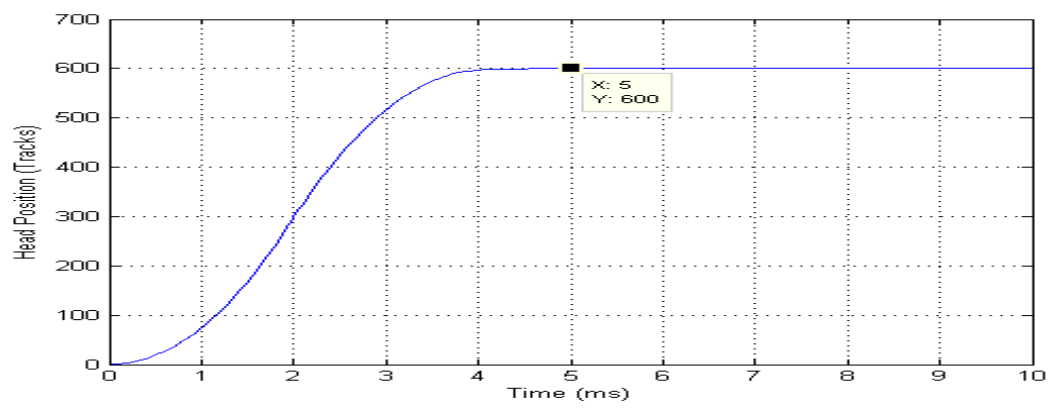

Fig 4. HDD head position using PTOS with state estimator and integral control in presence of the noise

Fig.5 represents the simulation result of PTOS with state estimator in presence of the noise that extended by bias estimator. It can be seen that the head reaches within $10 \%$ of the target position at 5 milliseconds same as obtained by PTOS with integral control.

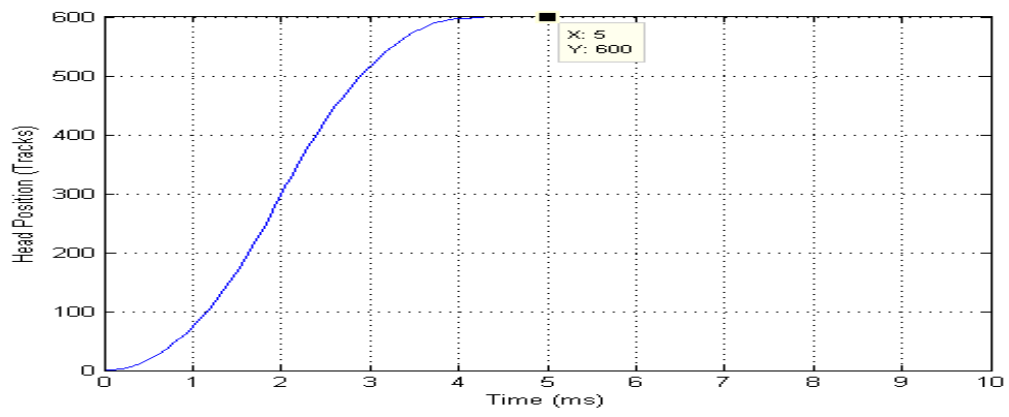

Fig 5. HDD head position using PTOS with state estimator and bias estimator in presence of the noise 


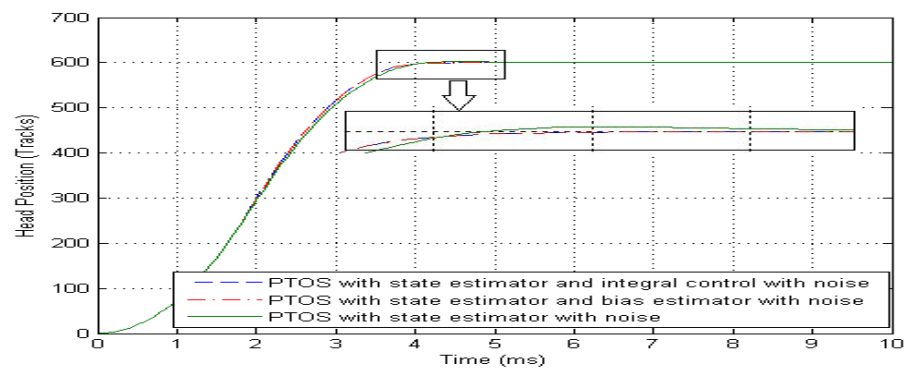

Fig 6. Comparison between HDD head position using PTOS with state estimator in presence of the noise, PTOS with state estimator and integral control in presence of the noise and PTOS with state estimator and bias estimator in presence of noise

\section{Conclusion}

Simulations are conducted on the system to compare the performances of the PTOS with integral control and PTOS with bias estimator in contrast to the PTOS. The difference between PTOS with state estimator, PTOS with state estimator and bias estimator and PTOS with state estimator and integral control in presence of the disturbance signals is shown in Fig.6. It is clear that PTOS with integral controller and bias estimator are more aggressive and their settling times are better about $0.8(\mathrm{~ms})$. Also, it is evident that HDD head position response using both PTOS with state estimator extended by integral control and PTOS with state estimator and bias estimator is same and both of them are able to eliminate the effect of input disturbance. But the order of estimator via bias estimator increases by 1 because the additional integrator is estimated by state estimator while the PTOS with integral control uses integrator as a new state in the feedback control and it is not estimated by state estimator, so the order of state estimator remain as before. By increasing in order of system, the steady state error becomes zero.

\section{References}

[1] P. A. Ioannou, E.B. Kosmatopoulos, A.M. Despain, "Position error signal estimation at high sampling rates using data and servo sector measurements " , IEEE Transactions on Control Systems Technology, Vol.11, issue.3, pp. 325-334, May 2003.

https://doi.org/10.1109/TCST.2003.810400

[2] A. S. I. Zinober and A. T. Fuller, "The sensitivity of nominally time optimal control systems to parameter variation," International Journal of Control, vol. 17, no. 4, pp. 673-703, April 1973.

https://doi.org/10.1080/00207177308932413

[3] E. P. Ryan, "On the sensitivity of a time-optimal switching function," IEEE Transaction on Automatic Control, vol. AC25, no. 2, pp. 275-277, April 1980.

https://doi.org/10.1109/TAC.1980.1102291

[4] A. A. Mamun, G.X. Guo and C. Bi, Hard Disk Drive: Mechatronics and Control. Boca Raton, Florida: CRC Press, 2007.

[5] A. Ebrahimi, S. A. A. Mousavian and M. Mirshams, "Near-Minimum Time Optimal Control of Flexible Spacecraft during Slewing Maneuver", K. N. Toosi University, Mechanical Dept., Iran, 2005.

[6] L. P. Lisboa, H. Lasevich, N. R. B. Neto, A.T. Salton and J.V. Flores, "Proximate Time Optimal Control of an XY Positioning Table", Embedded Systems (SASE/CASE), 2013 Fourth Argentine Symposium and Conference on, pp. 1-5, 2013.

https://doi.org/10.1109/sase-case.2013.6636773

[7] M. L. Workman, “Adaptive Proximate Time-Optimal Servomechanisms,” Ph.D. dissertation, Stanford Univ., Stanford, CA, 1987.

[8] M. L.Workman, R. L. Kosut, and G. F. Franklin, "Adaptive proximate time-optimal servomechanisms: Continuous time case," in Proc. American Control Conf., Minneapolis, MN, Jun. 1987, vol. 1, pp. 589-594.

[9] M. L.Workman, R. L. Kosut, and G. F. Franklin, "Adaptive proximate time-optimal servomechanisms: Discrete time case," in Proc. IEEE Conf. Decision and Control, Los Angeles, CA, Dec. 1987, vol. 2, pp. 1548-1553.

https://doi.org/10.1109/cdc.1987.272675 
[10] M. L.Workman and G. F. Franklin, "Implementation of adaptive proximate time-optimal controllers," in Proc. American Control Conf., Atlanta, GA, Jun. 1988, vol. 88, pp. 1629-1635.

[11] A. Dhanda and G. F. Franklin, "An Improved 2-DOF Proximate Time Optimal Servomechanism", IEEE Transaction on Magnetic, Vol. 45, no. 5, pp. 2151 - 2164, MAY 2009.

https://doi.org/10.1109/TMAG.2009.2013247

[12] M. Kalyon, S. Charifa, "Designing Continuous Proximate Time-Optimal Control System for a Class of Third-Order Systems," International Journal of Control and Automation, Vol. 4, no. 3, September 2011. 